\title{
Cateterismo intermitente limpo no paciente com lesão medular: conhecimento dos enfermeiros
}

\section{Clean intermittent catheterization in patients with spinal cord injury: knowledge of nurses \\ Cateterismo intermitente limpio en el paciente con lesión de la médula espinal: conocimiento de los enfermeros}

Roberta Silmara Miranda1, ", Gisela Maria Assis', Ana Claudia Lima Dornellas', Aline Maria Benedita Messias' Valeria Teles Batista ${ }^{1}$, João Júnior Gomes ${ }^{1}$

ORCID IDS

Miranda RS (DD https://orcid.org/0000-0003-4004-350X

Assis GM (iD https://orcid.org/0000-0001-6343-8075

Dornellas ACL (D) https://orcid.org/0000-0002-3349-214X

Messias AMB (D) https://orcid.org/0000-0002-3039-8211

Batista VT (D) https://orcid.org/0000-0001-8284-1484

Gomes JJ (D) https://orcid.org/0000-0003-4171-2752

\section{COMO CITAR}

Miranda RS; Assis GM; Dornellas ACL; Messias AMB; Batista VT; Gomes Jj. Cateterismo intermitente limpo no paciente com lesão medular: conhecimento dos enfermeiros. ESTIMA, Braz. J. Enterostomal Ther., 18: e0220, 2020. https://doi.org/10.30886/estima.v18.828_PT

\begin{abstract}
RESUMO
Introdução: A lesão medular resulta em falha no esvaziamento da bexiga, deixando o indivíduo exposto a risco de infecção recorrente de trato urinário, refluxo vesicoureteral e até perda da função renal. O cateterismo intermitente limpo (CIL) é o método de escolha para esvaziamento da bexiga nesses casos. Apesar de ter uma técnica simples, sua realização deve ser bem orientada a fim de evitar complicações como infecções ou traumas. A orientação para a técnica deve ser realizada ainda no período de internação pela lesão, cujo responsável é o enfermeiro. Objetivo: Avaliar o conhecimento dos enfermeiros que atuam em hospital de atendimento ao trauma com relação ao cateterismo intermitente limpo. Métodos: Questionário construído com base nas diretrizes da Associação Europeia de Enfermeiros Urológicos, aplicado a 18 enfermeiros de um hospital universitário, referência no atendimento do trauma raquimedular, a respeito de disfunção neurológica de trato urinário inferior e cateterismo intermitente limpo. Resultados: Os participantes apresentaram conhecimento expressivo a respeito da disfunção neurológica de trato urinário inferior e CIL. Houve erros quanto à técnica do CIL nos quesitos de indicação do uso luvas de procedimento, na lubrificação do cateter, coleta de culturas periódicas de urina, uso antibióticos e na necessidade de orientação antes da alta hospitalar. Conclusão: Apesar de a amostra demonstrar conhecimento em várias questões relacionadas ao tema, os erros indicam necessidade de capacitação e principalmente de conscientização quanto à responsabilidade de orientação antes da alta hospitalar.
\end{abstract}

DESCRITORES: Cateterismo urinário; Educação em saúde; Enfermeiro; Estomaterapia.

\begin{abstract}
Introduction: Spinal cord injury results in failure to empty the bladder, leaving the individual exposed to the risk of recurrent urinary tract infection, vesicoureteral reflux and even loss of renal function. Clean intermittent catheterization (CIC) is the method of choice for emptying the bladder in these cases. Although it has a simple technique, its performance should be well oriented in order to avoid complications such as infections or traumas. Guidance for the technique should be performed during the hospitalization period due to the injury and the nurse is responsible for this action. Objective: To evaluate the knowledge of nurses working in a trauma care hospital in relation to clean intermittent catheterization. Methods: Questionnaire constructed based on the guidelines of the
\end{abstract}

1.Faculdade de Medicina de São José do Rio Preto - Pós-Graduação em Enfermagem em Estomaterapia - São José do Rio Preto (SP), Brasil.

*Autor correspondente: mirandarobertas@gmail.com

Recebido: Abr. 12, 2019 | Aceito: Mar. 09, 2020 
European Association of Urological Nurses, applied to 18 nurses from a university hospital, a reference in the treatment of spinal trauma, regarding neurological dysfunction of the lower urinary tract and clean intermittent catheterization. Results: The participants presented expressive knowledge about lower urinary tract neurological dysfunction and $\mathrm{CIC}$. There were errors regarding the CIC technique in the indication of the use of procedure gloves, in the lubrication of the catheter, collection of periodic urine cultures, use of antibiotics and in the need for instructions before discharge from hospital. Conclusion: Although the sample demonstrated knowledge on several issues related to the subject, the errors indicate the need for training and especially awareness of the responsibility of guidance before discharge from hospital.

DESCRIPTORS: Urinary catheterization; Health education; Nurse; Enterostomal therapy.

\section{RESUMEN}

Introducción: La lesión de la médula espinal provoca la imposibilidad de vaciar la vejiga, dejando al individuo expuesto al riesgo de infección recurrente del tracto urinario, reflujo vesicoureteral e incluso pérdida de la función renal. El cateterismo intermitente limpio es el método de elección para vaciar la vejiga en estos casos. A pesar de tener una técnica simple, su rendimiento debe estar bien orientado para evitar complicaciones como infecciones o traumatismos. La guía para la técnica debe realizarse durante el período de hospitalización debido a la lesión y la enfermera es responsable de esta acción. Objetivo: Evaluar el conocimiento de las enfermeras que trabajan en un hospital de atención de traumas en relación con la cateterización intermitente limpia. Métodos: Cuestionario construido sobre la base de las directrices de la Asociación Europea de Enfermeras Urológicas, aplicado a 18 enfermeras de un Hospital Universitario, una referencia en el tratamiento del trauma espinal, con respecto a la disfunción neurológica del tracto urinario inferior y el cateterismo intermitente limpio. Resultados: Los participantes presentaron conocimiento expresivo sobre la disfunción neurológica del tracto urinario inferior y el cateterismo intermitente limpio. Hubo errores con respecto a la técnica CIL en términos de indicar el uso de guantes de procedimiento, en la lubricación del catéter, la recolección de urocultivos periódicos, el uso de antibióticos y la necesidad de orientación antes del alta hospitalaria. Conclusión: Aunque la muestra demuestra conocimiento sobre varios temas relacionados con el tema, los errores indican la necesidad de capacitación y especialmente la conciencia de la responsabilidad de la orientación antes del alta hospitalaria.

DESCRIPTORES: Cateterismo urinario; Educación sanitaria; Enfermería; Estomatoterapia.

\section{INTRODUÇÃO}

O enfermeiro sente a cada dia a necessidade de desenvolver seu conhecimento científico em busca de respostas aos problemas de saúde com o intuito de alcançar a excelência na prestação de cuidados ${ }^{1}$. Um meio eficaz de atingir esse objetivo é a educação em saúde, que proporciona a ampliação dos conhecimentos, com perspectivas de ultrapassar dificuldades e conquistar maior autonomia ${ }^{2}$. Existem condições de saúde especialmente desafiadoras para a atuação do enfermeiro, e a lesão medular é uma delas. Trata-se de um evento avassalador na vida do ser humano que provoca prejuízo às estruturas do canal medular levando a alterações nas funções motoras, sensitivas e autonômicas, com consequentes alterações no padrão miccional, nas fases de enchimento e esvaziamento urinário. As lesões medulares são causadas predominantemente por traumas em acidentes automobilísticos, ferimentos por armas de fogo e quedas ${ }^{3-4}$.

Voltando a citar o sistema urinário, na lesão medular ocorre uma interrupção na comunicação entre este e o sistema nervoso central. Essa condição é definida como disfunção neurológica de trato urinário inferior (DNTUI) e pode se manifestar como bexiga hiperativa, com contrações involuntárias na fase de armazenamento da urina, ou hipoativa, com distensão vesical e transbordamento, ambas com risco de infecção urinária e refluxo vesicoureteral ${ }^{5}$.

A DNTUI resulta frequentemente em resíduo pós-miccional (RPM) elevado, por esse motivo requer cateterização para esvaziamento completo da bexiga de forma periódica. O cateterismo intermitente limpo (CIL) é a primeira técnica de escolha. Sua técnica desobriga uso de luvas estéreis e antissépticos e pode ser empregada por pacientes ou cuidadores em ambiente domiciliar ${ }^{6}$. O procedimento de CIL foi relatado em publicações científicas pela primeira vez por Lapides et al., em 1972, que demonstraram que a infecção de trato urinário (ITU) nas DNTUI é causada por RPM e distensão vesical, não necessariamente pela assepsia da técnica, provando assim ser mais importante manter uma regularidade de esvaziamento do que uma técnica asséptica ${ }^{7}$.

Os passos a serem seguidos são a higiene da região genital, das mãos, lubrificação e introdução do cateter ${ }^{8}$. A independência na realização do procedimento pode não ser alcançada por pacientes com lesões altas, porém a possibilidade de melhoria da função motora, ou seja, melhora da mobilidade das mãos surge após o primeiro ano de alta 
quando há acompanhamento e aconselhamento do paciente sobre o controle da bexiga?.

Apesar de ser o método de escolha para esvaziamento da bexiga e prevenção de complicações na DNTUI, o CIL não é isento de complicações. Mesmo com uma técnica relativamente simples, ele precisa ser realizado de maneira sistematizada; para isso, o paciente deve ser capacitado pelo enfermeiro ainda no período de internação ${ }^{10}$. Segundo as diretrizes do Ministério da Saúde de atenção à pessoa com lesão medular, todo paciente com lesão medular precisa ser orientado para o CIL antes da alta hospitalar independentemente do estudo urodinâmico. Portanto é essencial que os enfermeiros que assistem esses pacientes, estejam capacitados para dar tais orientações ${ }^{3}$.

O processo educativo a respeito do CIL é de grande importância. A possibilidade de um treinamento no âmbito hospitalar favorece as observações das aceitações e rejeições do paciente, facilita o conhecimento do seu próprio corpo, localização da uretra, a importância dos cuidados de higiene pessoal, entre outros aspectos ${ }^{11}$.

Diante do cenário apresentado e da vivência das pesquisadoras, delineou-se o seguinte objetivo: identificar se os enfermeiros que atuam em hospitais que prestam atendimento à pessoa com lesão medular estão preparados para orientar o CIL. Espera-se com a publicação dos resultados lançar luz à temática de forma a motivar a elaboração de protocolos assistenciais que garantam ao paciente uma capacitação precoce e de qualidade, no que diz respeito ao cateterismo intermitente limpo de forma a evitar complicações.

\section{MÉTODO}

Estudo exploratório descritivo de corte transversal com abordagem quantitativa. A pesquisa foi conduzida em um hospital universitário do estado de Minas Gerais. Participaram da pesquisa enfermeiros que atuavam nas unidades de internação que admitem pacientes com trauma raquimedular. A amostra foi não probabilística, por conveniência, o recrutamento foi realizado através da escala de trabalho fornecida pelo enfermeiro responsável, o enfermeiro participante foi convidado presencialmente no dia da coleta de dados, no horário de trabalho. Foram excluídos os enfermeiros que estavam de férias, licença médica, licença maternidade e atividades das quais não podiam se ausentar no momento da aplicação do questionário de avaliação da pesquisa.
A coleta de dados foi realizada no período de 6 a 22 de fevereiro de 2019, após aprovação do projeto pelo Comitê de Ética e Pesquisa com Seres Humanos, com parecer $\mathrm{n}^{\circ}$ 3.114.560. As pesquisadoras foram até os setores selecionados após conversa com o enfermeiro responsável e abordaram presencialmente cada enfermeiro com critérios de elegibilidade para o estudo. Foram explicados detalhadamente todos os aspectos éticos e técnicos da pesquisa e o questionário do estudo foi aplicado somente mediante assinatura do Termo de Consentimento Livre e Esclarecido. O questionário foi elaborado pelas pesquisadoras para esta pesquisa baseado em diretrizes internacionais. O instrumento foi composto por dados de caracterização da amostra e questões verdadeiras e falsas a respeito dos passos e cuidados na realização da técnica. As variáveis quantitativas foram descritas por média, desvio padrão, valor mínimo e valor máximo. Variáveis categóricas foram descritas por frequência absoluta e relativa.

A análise foi feita através de frequências absolutas e relativas e cruzamentos dos dados de conhecimento de acerto e erro para cada item sobre conhecimento do enfermeiro a respeito do CIL, com as variáveis de identificação como idade, formação, tempo e local de atuação. Todas as análises foram feitas com auxílio do programa estatístico Statistical Package for Social Sciences (SPSS), versão 24.0.

\section{RESULTADOS}

Participaram do estudo 18 enfermeiros. A Tabela 1 apresenta os dados de perfil da amostra. Nota-se que a amostra foi composta predominantemente por mulheres, com idade média de 37,6 anos, com curso de pós-graduação, e média de 11 anos de atuação como enfermeiras.

A Tabela 2 apresenta a frequência e o percentual de respostas corretas assinaladas pelos enfermeiros para cada conteúdo abordado a respeito do cateterismo intermitente limpo.

Observa-se que grande parte dos enfermeiros tem conhecimento de que a lesão medular resulta em bexiga hipoativa ou hiperativa, e que as duas podem levar à retenção urinária crônica. Apesar desse resultado, um pequeno percentual da amostra indicou que o CIL deve ser orientado antes da alta hospitalar e mais da metade dos participantes citou que ele deve ser ensinado apenas após o resultado do estudo urodinâmico. 
Tabela 1. Perfil da amostra.

\begin{tabular}{|c|c|c|}
\hline Variável & Classificação & Resultado* \\
\hline Idade (anos) & & $37,6 \pm 7,5(23-52)$ \\
\hline \multirow{2}{*}{ Sexo } & Feminino & $14(77,8)$ \\
\hline & Masculino & $4(22,2)$ \\
\hline \multirow{3}{*}{ Curso de pós-graduação } & Não & $5(27,8)$ \\
\hline & Sim & $13(72,2)$ \\
\hline & Clínica cirúrgica 1 & $7(38,9)$ \\
\hline \multirow[t]{2}{*}{ Setor de atuação } & Clínica cirúrgica 2 & $6(33,3)$ \\
\hline & Clínica cirúrgica 3 & $5(27,8)$ \\
\hline
\end{tabular}

*Descritos por média \pm desvio padrão (mínimo - máximo)

Tabela 2. Frequência e percentual de respostas corretas pelos enfermeiros a respeito do cateterismo intermitente limpo.

\begin{tabular}{|c|c|}
\hline Pergunta & Resultado n (\%) \\
\hline Consequências da lesão medular & $13(72,2)$ \\
\hline Orientação do CIL antes da alta & $7(38,9)$ \\
\hline Indicação do CIL após exame urodinâmico & $10(55,6)$ \\
\hline Técnica pelo cuidador realizada sem toque & $15(83,3)$ \\
\hline Realização da técnica sem infraestrutura & $13(72,2)$ \\
\hline Higiene das mãos com água corrente e sabonete & $16(88,9)$ \\
\hline Uso de álcool gel para higiene das mãos & $14(77,8)$ \\
\hline Uso de luvas estéreis & $16(88,9)$ \\
\hline Uso de luvas de procedimento & $6(33,3)$ \\
\hline Técnica sem toque pelo cuidador & $14(77,8)$ \\
\hline Uso de antissépticos na higiene genital & $11(61,1)$ \\
\hline Higiene genital com água e lenço umedecido & $12(66,7)$ \\
\hline Dermatites por antissépticos & $13(72,2)$ \\
\hline Gel hidrossolúvel para lubrificação & $13(72,2)$ \\
\hline Injeção de lidocaína gel & $9(50,0)$ \\
\hline Lubrificação com produtos oleosos & $17(94,4)$ \\
\hline Extensão de lubrificação do cateter homem/mulher & $6(33,3)$ \\
\hline Introdução lenta até iniciar drenagem de urina & $14(77,8)$ \\
\hline Ângulo do pênis para introdução & $15(83,3)$ \\
\hline Forçar a introdução em caso de resistência & $16(88,9)$ \\
\hline Pressão suprapúbica pré-cateterismo & $12(66,7)$ \\
\hline Calibre do cateter (08 a $12 \mathrm{Fr}$ ) & $12(66,7)$ \\
\hline Reutilização do cateter & $16(88,9)$ \\
\hline Indicação de uso único & $17(94,4)$ \\
\hline Frequência ideal de realização da técnica & $13(72,2)$ \\
\hline Importância do diário vesical na definição de frequência & $18(100)$ \\
\hline Suspensão do CIL se presença de diurese espontânea & $16(88,9)$ \\
\hline Redução de intervalos se perda urinária & $15(83,3)$ \\
\hline Redução de ingestão de água & $18(100)$ \\
\hline Urocultura e antibioticoterapia & $4(22,2)$ \\
\hline
\end{tabular}


A maioria dos enfermeiros acertou que o CIL pode ser realizado por um cuidador comprometido em manter a frequência recomendada do procedimento, e que deve ser realizado mesmo que falte infraestrutura como pias e vasos sanitários. A higiene das mãos também foi um item de grande percentual de acerto, a maioria indicou que a recomendação é de água corrente e sabonete líquido e que o álcool em gel não deve substituir a higiene completa.

Grande parte dos respondentes acertou que não há indicação de uso de luvas estéreis, porém a maioria errou não indicando a realização da técnica sem luvas de procedimento, mesmo com as mãos higienizadas. Quanto à indicação de técnica sem toque pelo cuidador, utilizando luvas ou gaze, a maioria acertou que essa é a melhor recomendação.

As questões de higiene genital tiveram um grande percentual de acertos. A maioria assinalou que não existe a indicação de uso de antissépticos, que água ou lenço umedecido são as opções para todas as cateterizações e que o uso frequente de antissépticos pode levar a dermatites locais. Quanto à lubrificação do cateter, a maioria acertou que deve ser com gel hidrossolúvel e que lubrificantes oleosos não são indicados; houve erros no sentido de não desencorajar a injeção de lidocaína na uretra e também na extensão de lubrificação do cateter para homens e mulheres.

Houve percentual expressivo de respostas corretas com relação à introdução lenta do cateter, à angulação do pênis, a não forçar a passagem do cateter e não fazer pressão suprapúbica antes do cateterismo. Os dados apresentaram comportamento semelhante nos itens de calibre do cateter, não indicação de reutilização do cateter.

Os enfermeiros também responderam predominantemente de forma correta quanto à frequência ideal para o cateterismo, à importância do diário vesical para definição de frequência, à não suspensão do procedimento na presença de micção espontânea e à redução do intervalo em casos de perdas urinárias frequentes.

Todos os respondentes acertaram que não há indicação de reduzir ingestão de água e a maioria errou no sentido de indicar urocultura periódica e tratamento com antibioticoterapia em casos de bacteriúria.

As variáveis de caracterização da amostra foram cruzadas com as variáveis de conhecimento a respeito do tema. Para a comparação dos dois grupos, com relação a variáveis quantitativas, foi usado o teste $t$ de Student para amostras independentes ou o teste não paramétrico de Mann-Whitney. As variáveis categóricas foram analisadas considerando-se o teste exato de Fisher. Para avaliação da associação entre duas variáveis quantitativas foram estimados coeficientes de correlação de Spearman, valores de $\mathrm{p}<0,05$ indicaram significância estatística. Não houve associação significativa entre o acerto nas respostas e as variáveis sexo, setor de atuação, tempo de formação e pós-graduação.

\section{DISCUSSÃO}

O perfil da amostra estudada é condizente com o perfil da enfermagem brasileira, demonstrada também por outros estudos com relação a sexo predominante, idade média e formação ${ }^{12,13}$.

A questão de consequências urológicas da lesão medular teve bom percentual de acerto, demonstrando que os enfermeiros sabem que os pacientes atendidos terão o padrão miccional alterado. As alterações urológicas provocadas pela lesão na medula espinhal são umas das maiores inquietações dos profissionais da reabilitação, porque o funcionamento vesical inadequado, quando tratado incorretamente, causa condições desfavoráveis como infecção urinária, cálculos vesicais, fístulas penoescrotais, refluxo vesicoureteral, hidronefrose e pode levar até a perda da função renal ${ }^{3}$.

Um dado intrigante, talvez o mais preocupante, foi com relação à orientação do CIL pré-alta. Poucos acertaram que é obrigatória, mesmo tendo acertado que os pacientes apresentarão importantes disfunções a partir da lesão medular. Nesse aspecto, as Diretrizes de Atenção à Pessoa com Lesão Medular indicam que se institua o CIL de forma mandatória, independentemente da realização precoce do exame de urodinâmica, desde a alta hospitalar, e a orientação do paciente ou cuidador antes da alta ${ }^{3}$.

Quanto à realização da técnica por um cuidador, predominantemente com respostas corretas pela amostra, a Sociedade Brasileira de Urologia e a Associação Europeia de Enfermeiros Urológicos enfatizam a necessidade de treinar os pacientes, familiares e cuidadores sobre o procedimento correto do cateterismo intermitente limpo a fim de evitar complicações. Nos casos de pacientes sem agilidade manual, por perdas de movimentos e falta de adaptação aos dispositivos auxiliares ou por dificuldade de aprendizagem, a presença de um cuidador para ser treinado a executar a técnica é imprescindível ${ }^{14}$. Pesquisa aplicada em hospital do interior de São Paulo com 141 usuários de CIL apresentou em seu resultado que metade desses pacientes necessitam de ajuda 
de familiares, cuidadores ou profissionais de saúde da atenção primária para executar o procedimento ${ }^{15}$.

Grande parte da amostra acertou que o CIL deve ser realizado mesmo sem infraestrutura, para manter a sua regularidade ideal e não ocorrer armazenamento de urina com volume superior a $500 \mathrm{ml}$. Os usuários de CIL devem ser atentos à questão da infraestrutura, no entanto não devem deixar de fazer o CIL devido à falta de local limpo, por estar fora de casa ou em uma viagem; porque o cateterismo regular atua na prevenção de infecções e demais consequências relacionadas à diurese estagnada ${ }^{16}$.

Com relação à higiene das mãos com água e sabão, não substituição por álcool em gel e não necessidade de luvas estéril, a maioria respondeu de forma correta e mostrou conhecer o assunto, porém no tópico uso de luvas de procedimento, a maioria respondeu ser necessário, o que pode onerar e dificultar o procedimento. A recomendação para a higienização das mãos com água e sabão, de preferência sabonete líquido, é citada por diversos autores ${ }^{6,8,14} . \mathrm{Na}$ ausência de infraestrutura como pias, orientam a levar água em uma garrafa ou outro utensílio para lavar as mãos e o meato uretral ${ }^{8}$. Há profissionais que indicam o uso do lenço umedecido na higienização da uretra, como alternativa na falta de outros recursos ${ }^{14}$. Não foram encontradas referências que indicassem a utilização do álcool em gel em substituição à água e sabão na técnica do CIL, mas vale considerar a forte campanha realizada pelos serviços de controle de infecção hospitalar, garantindo sua ação na ausência de sujidade e com a necessidade de fricção. $O$ manual de referência técnica para higiene das mãos não relacionada ao CIL cita que a higiene pode ser realizada friccionando as mãos com solução alcoólica ou com água e sabonete, sendo as duas técnicas capazes de deixar o paciente livre de contaminações da microbiota ${ }^{17}$.

Quanto ao uso de luvas, as estéreis são recomendadas para técnica asséptica em ambiente hospitalar.Já as luvas de procedimento podem ser usadas em ambiente domiciliar. Seu uso, porém,é dispensável na técnica limpa, mantendo-se a higienização prévia das mãos. A única exceção em que o cateterismo intermitente limpo deve ser realizado com rigor da técnica estéril é em pacientes neuropáticos imunossuprimidos ${ }^{14,15}$.

Os participantes acertaram que a técnica sem toque pode ser realizada pelo cuidador. A técnica sem toque consiste na introdução do cateter sem tocar na porção que entrará em contato com a bexiga. É a técnica mais indicada e os cateteres prontos para uso (lubrificados e hidrofílicos) são os mais adequados para a execução ${ }^{6}$. Revisão integrativa de artigos sobre as melhores práticas de CIL, realizada no período de 2010 a 2015, demonstrou que o procedimento de CIL com a técnica de não toque reduz a probabilidade de infecção do trato urinário comparado com o cateterismo intermitente estéril ${ }^{18}$.

Não existe indicação do uso de antisséptico para a higiene genital antes do CIL e mais da metade dos participantes respondeu corretamente. Estudos trazem que o uso de antissépticos causa dermatites, o que vai ao encontro da resposta dos enfermeiros pesquisados. A forma mais correta de higiene da uretra é a limpeza com uma gaze molhada com água, se não há sujidade visível, ou lenço umedecido realizando movimentos circulares por duas ou três vezes, antes da introdução do cateter, lembrando que a região genital deve ser higienizada com água e sabão duas vezes ao dia no horário do cateterismo; e mais da metade da amostra também respondeu de forma correta ${ }^{6,8,19}$. Revisão sistemática com metanálise buscou comparar a eficácia da limpeza do meato uretral antes do cateterismo vesical com água ou solução salina e antissépticos e comprovou que não houve diferença estatisticamente significativa quando comparados os dois métodos no quesito de prevenção de ITU. Portanto sugeriram revisão de diretrizes para reduzir custos e prevenir alergias que podem ser causadas pelos antissépticos ${ }^{20}$.

A maioria dos pesquisados assinalaram como correto o uso de géis hidrossolúveis para a lubrificação antes do CIL, concordando com a literatura que indica o uso de lubrificação com gel em cateteres sem revestimento, os lubrificantes indicados são os géis hidrossolúveis. Na técnica limpa não é necessário o uso de géis estéreis ${ }^{8,14,21}$. A literatura indica o uso de lubrificantes hidrofilicos, portanto o uso de produtos oleosos é contraindicado. Produtos oleosos, por serem hidrofóbicos, se desprendem do cateter quando entram em contato com a urina e deixam resíduos dentro da bexiga, favorecendo assim a formação de cálculos vesicais ${ }^{8}$.

Metade dos entrevistados erraram ao assinalar que a injeção de lidocaína antes do cateterismo é correta. A injeção de lidocaína não é indicada devido ao uso frequente, que pode resultar em absorção sistêmica ${ }^{6}$, além de mobilizar a flora microbiana distal para a porção proximal da uretra. Revisão integrativa de literatura a respeito da aplicação da lidocaína 2\% no cateterismo urinário masculino demonstrou que não foi observada diferença estatística significante quanto à instilação ou não de lidocaína intrauretral. Também não demonstrou que a atividade analgésica da lidocaína seja 
eficiente, e não há consenso de quanto tempo deve-se aguardar para introduzir o cateter ou se ele deve ser introduzido de imediato. Existem lacunas na literatura sobre o tema, sendo sugeridos novos estudos ${ }^{21}$.

Embora não existam publicações a respeito, é de consenso dos profissionais não indicar a injeção de lidocaína no CIL por dois motivos. O primeiro é o deslocamento frequente da flora distal da uretra para região proximal. O segundo é relacionado à lidocaína propriamente dita, pois não se sabe qual seria a absorção pela mucosa uretral, considerando o grande número de procedimentos ao longo da vida. Razões que justificam a ausência da orientação de injeção de lidocaína no CIL em consensos e diretrizes.

A maioria dos participantes acertou a questão quanto à introdução lenta do cateter, angulação do pênis e contraindicação de forçar a introdução em caso de resistência. A introdução lenta do cateter bem como evitar forçar a passagem se encontrar resistência são cuidados essenciais para prevenir traumas e o desenvolvimento de estenoses uretrais ${ }^{14}$. $\mathrm{O}$ aumento da próstata ou a contração involuntária do esfíncter podem causar resistência, no caso da próstata, e uma leve redução de angulação do pênis pode ajudar; quanto à contração esfincteriana, deve-se mante o cateter na mesma posição, esperando seu relaxamento ${ }^{8,14}$.

Grande parte da amostra acertou a contraindicação da pressão abdominal ou compressão suprapúbica précateterismo. Análise retrospectiva de prontuários de pacientes com espinha bífida e bexiga hipoativa mostrou que a micção por Valsalva é prejudicial devido à alta pressão da bexiga e alta pressão aplicada ao períneo com esforço abdominal ${ }^{22}$ Pesquisa qualitativa e descritiva aplicada em familiares de crianças com bexiga neurogênica e em uso de cateterismo intermitente limpo observou a adoção de técnicas inadequadas como micção por massagem abdominal ${ }^{23}$.

Quanto ao calibre ideal do cateter, ele deve ter o diâmetro suficiente para permitir o fluxo livre de urina em um tempo aceitável, com o menor calibre possível para não provocar lesões na uretra conforme diretriz da Associação Europeia de Enfermeiros em Urologia ${ }^{6}$. Os participantes assinalaram acertivamente a indicação de calibres de 8 a 12 Fr para o adulto.

A reutilização do cateter não é recomendada e a maioria dos enfermeiros respondeu essa afirmação corretamente. Uma revisão de literatura a respeito de ITU e os custos relacionados ao uso único ou reutilização de diferentes cateteres, no período de 2014 a 2018, confirmou que não existem pesquisas científicas suficientemente seguras para orientar a reutilização de cateteres.
A reutilização de cateteres expõe o usuário a técnicas de higiene do cateter que não foram testadas em pesquisas consistentes. O tempo de reutilização do cateter também não foi definido ${ }^{24}$. Autores de outra revisão bibliográfica consideraram a orientação de uso de cateter único a cada procedimento como medida de prevenção de infecção ${ }^{16}$. Reforçam que faltam pesquisas satisfatórias sobre um método ideal de limpeza de cateteres de uso múltiplo, razão da não recomendação ${ }^{16,24}$.

Grande porcentagem de enfermeiros pesquisados respondeu que a frequência ideal de realização da técnica deve ser mantida e apontaram para a importância do diário vesical para definição desses intervalos. O profissional de saúde especializado deve indicar o número de cateterismos a ser executado em 24 horas conforme informações sobre a ingestão diária de líquidos e volume de urina drenado, dados extraídos do diário vesical. $\mathrm{O}$ volume de diurese a cada procedimento não deve ultrapassar $400 \mathrm{ml}$, volumes superiores levam a risco de ITU por distensão ${ }^{6,16}$.

Não é aconselhada a redução de ingestão de água para que não ocorram mudanças na cor e no odor da urina. Todos os participantes concordaram assertivamente com essa afirmação. A ingestão hídrica é considerada uma das melhores práticas para prevenção de infecção do trato urinário ${ }^{16}$. Se a hidratação é eficiente, a urina é diluída e mantém diurese constante. $\mathrm{O}$ volume de ingestão diária indicado é $25-35 \mathrm{ml} / \mathrm{kg}$. A ingesta abusiva de líquidos, por outro lado, aumenta a chance de hiperdistensão da bexiga e transbordamento, sinalizados pela incontinência. A pessoa com DNTUI pode apresentar perda urinária por transbordamento ou hiperatividade detrusora. A presença de diurese espontânea não indica possibilidade de suspensão do CIL, questão acertada por grande parte dos participantes.

Pequena parte dos pesquisados acertaram que não é indicado urocultura periódica e terapêutica com antibióticos, se bacteriúria. Os usuários de CIL apresentam o trato urinário colonizado, portanto uroculturas tendem a ser positivas. A ITU deve ser investigada por sinais e sintomas que os pacientes devem ser orientados a reconhecer. A cultura nesses casos serve apenas para guiar a escolha do tratamento medicamentoso ${ }^{3,6,14}$.

\section{CONCLUSÃO}

A amostra estudada apresentou conhecimento considerável a respeito de disfunção neurológica urinária de trato urinário inferior e do cateterismo intermitente 
limpo. Questões a respeito da indicação do procedimento sem luvas, não necessidade de urocultura periódica e tratamento para bacteriúria assintomática, contraindicação de injetar lidocaína na uretra de forma regular e da quantidade de lubrificante no cateter para a inserção foram itens em que menos da metade da amostra apresentou acerto, indicando necessidade de atividades de atualização.

A resposta com grande número de erros que se mostrou mais preocupante foi com relação à orientação do paciente para CIL antes da alta. A maioria da amostra não assinalou essa afirmativa como verdadeira, sendo que o local da amostra é referência no atendimento à lesão medular e todo enfermeiro deveria estar ciente e seguro para a orientação durante o período de internação.

Faz-se necessária a discussão do tema entre serviços que prestam tais atendimentos a fim de lançar luz da necessidade de programas de capacitação e implementação de protocolos assistenciais.

\section{AGRADECIMENTOS}

Agradecimentos à Faculdade de Medicina de São José do Rio Preto e ao Hospital das Clínicas da Universidade Federal de Uberlândia.

\section{CONTRIBUIÇÃO DOS AUTORES}

Miranda RS, Assis GM, Dornellas ACL, Messias AMB, Batista VT, e Gomes JJ; Conceitualização, Assis GM, Miranda RS, Dornellas ACL, Messias AMB, Batista VT, e Gomes JJ; Metodologia, Assis GM e Batista VT; Coleta de dados, Miranda RS; Redação- Primeira versão, Assis GM, Miranda RS, Dornellas ACL, Messias AMB e Batista VT; Redação - Revisão \& Edição, Assis GM, Miranda RS, Dornellas ACL, Messias AMB e Batista VT; Supervisão, Assis GM e Gomes JJ.

\section{REFERÊNCIAS}

1. Antônio V. Importância do enfermeiro especialista em enfermagem de reabilitação na promoção do autocuidado eliminação vesical à pessoa/família com paraplegia resultante de lesão vertebro medular. Santarém. Dissertação [Mestrado Enfermagem de Reabilitação] - Instituto Politécnico de Santarém, Escola Superior de Saúde; 2018.

2. Sousa LB, Torres CA, Pinheiro PNC, Pinheiro AKB. Práticas de educação em saúde no Brasil: a atuação da enfermagem. Rev Enferm UERJ. 2010;18(1):55-60.

3. Brasil. Ministério da Saúde. Diretrizes de atenção à pessoa com lesão medular. $2^{a}$ ed. Brasília (DF): Ministério da Saúde; 2015.

4. Dorsher PT, McIntosh PM. Neurogenic bladder. Adv Urol. 2012;2012(816274):1-16. https://doi.org/10.1155/2012/816274

5. McKibben MJ, Seed P, Ross SS, Borawski KM. Urinary tract infection and neurogenic bladder. Urol Clin North Am. 2015;42(4):527-36. https://doi.org/10.1016/j.ucl.2015.05.006

6. Vahr S, Cobussen-Boekhorst H, Eikenboom J, Geng V, Holroyd $\mathrm{S}$, et al. Evidence based guidelines for best practice in urological heath care. Catheterisation urethral intermittent in adults. Dilatation, urethral intermittent in adults. Arnhem: European Association of Urology Nurses; 2013.

7. Lapides J, Diokno AC, Silber SJ, Lowe BS. Clean, intermittent self-catheterization in the treatment of urinary tract disease. J Urol. 1972;107(3):458-61. https://doi.org/10.1016/S00225347(17)61055-3

8. Assis GM, Fraga R. Cateterismo intermitente limpo. Manual ilustrado de orientação ao usuário (adulto). Curitiba: Universidade Federal do Paraná - Hospital de Clínicas; 2015.
9. ElliottCS, Zlatev D, CrewJ, Shem K. Do appreciable changes in the upper extremity motor capability to perform clean intermittent catheterization come about with time after traumatic spinal cord injury? Neurourol Urodynam. 2019;38(3):975-80. https:// doi.org/10.1002/nau.23943

10. Alavinia SM, Omidvar M, Farahani F, Bayley M, Zee J, Craven BC. Enhancing quality practice for prevention and diagnosis of urinary tract infection during inpatient spinal cord rehabilitation. J Spinal Cord Med. 2017;40(6):803-12. https:// doi.org/10.1080/10790268.2017.1369216

11. Garcia F, Altamirano B, Machado C. Vaciado vesical a traves del cateterismo intermitente limpio. Medicina Infantil. 2016;23(2):192-3.

12. Araújo MAN, Lunardi Filho WDD, Alvarenga MRM, Oliveira RD, Souza JC, Vidmantas S. Sociodemographic profile of nurses of the hospital network. J Nurs UFPE on line. 2017 Nov;11(Supl. 11):4716-25.

13. Machado MH, Aguiar Filho W, Lacerda WF, Oliveira E, Lemos W, Wermelinger $\mathrm{M}$, et al. Características gerais da enfermagem: o perfil sócio demográfico. Enferm Foco. 2015;7:9-14. https:// doi.org/10.21675/2357-707X.2016.v7.nESP.686

14. Truzzi JC, Canalini AF, Prezotti JA, Resplande J. Recomendações SBU 2016. Cateterismo vesical intermitente. Rio de Janeiro (RJ): Sociedade Brasileira de Urologia; 2016.

15. Santos RCR, Fumincelli L, Nassiff A, Souza Júnior VD, Jorge BM, Mazzo A. Neurogenic bladder patient: intermittent urinary catheterization and intestinal care. J Nurs UFPE on line. 2015;9(Supl. 7):8953-60. 
16. Lamin E, Newman DK. Clean intermittent catheterization revisited. Int Urol Nephrol. 2016;48(6):931-9. https://doi. org/10.1007/s11255-016-1236-9

17. World Health Organization (WHO). Manual de referência para técnica para a higiene das mãos. Genebra: WHO; 2009.

18. Amorin RT, Cavalcante TB, Diniz BPO, Miranda SM, Sousa ARA, Rocha AJSC. Cateterismo uretral intermitente em ambiente hospitalar: revisão integrativa. In: XII Congresso Brasileiro de Estomaterapia 2017; 2017 nov. 12-15; Belo Horizonte (MG). Belo Horizonte: Sobest; 2017.

19. Brasil. Governo do Distrito Federal. Secretaria de Estado de Saúde. Portaria SES-DF nº 791 de 26 de julho de 2018. Protocolo de Atenção à Saúde: Atendimento ao Usuário com Necessidade de Cateterismo Vesical. Brasília (DF): Diário Oficial do Distrito Federal 7 ago. 2018; 6-7 [citado em 19 nov 2019]. Disponível em: http://uww.buriti.df.gov.br/ftp/diariooficial/2018/08_Agosto/ DODF\%20149\%2007-08-2018/DODF\%20149\%2007-082018\%20INTEGRA.pdf

20. Cunha M, Santos E, Andrade A, Jesus R, Aguiar C, Marques F, et al. Eficácia da limpeza ou desinfeção do meato urinário antes da cateterização urinária: revisão sistemática. Rev Esc Enferm USP. 2013;47(6):1407-13. https://doi.org/10.1590/S0080623420130000600023

21. Santos RP, Tres DP, Rosin J, Peres RR, Carvalho ARS. Aplicação lidocaína gel a 2\% no cateterismo urinário: revisão integrativa de literatura. Revista Varia Scientia - Ciências da Saúde. 2016;2(2):165-73.

22. El Akri M, Brochard C, Hascoet J, Jezequel M, Alimi Q, Khene Z-E, et al. Risk of prolapse and urinary complications in adult spina bifida patients with neurogenic acontractile detrusor using clean intermittent catheterization versus Valsalva voiding. Neurourol Urodynam. 2019;38(1):269-77. https://doi.org/10.1002/nau.23844

23. Antonio S, Pacheco STA, Gomes MPF, Reis AT, Rodrigues BMRD, Souza SM. Cateterismo intermitente limpo em crianças com bexiga urinária neurogênica: o cuidado do familiar no domicílio. Rev Enferm UERJ. 2015;23(2):191-6. https://doi.org/10.12957/ reuerj.2015.16493

24. Saadat SH, Shepherd S, Asseldonk BV, Elterman DS. Clean intermittent catheterization: single use vs. reuse. Can Urol Assoc J. 2019;13(2):64-9. https://doi.org/10.5489/cuaj.5357 